\title{
Editorial: Speed and Efficiency in Pharmaceutical Development
}

\author{
Stephen Scypinski
}

Published online: 18 August 2009

(C) International Society for Pharmaceutical Engineering 2009

Welcome to the first thematic issue of the Journal of Pharmaceutical Innovation (JPI) entitled "Speed and Efficiency in Pharmaceutical Development." At its 2007 meeting, the Editorial Advisory Board decided to devote one issue per year to a focused topic of general interest. The result of that discussion is the issue that you see before you, dedicated to the process of pharmaceutical development. It includes both research articles as well as perspectives that illustrate approaches to increasing productivity during the development and commercialization process for new pharmaceutical drug products.

It is well known that today's economic and regulatory climate demands the implementation of new and innovative ways to bring medicines to treat unmet medical needs to market faster and cheaper without compromising quality. The increased scrutiny by regulatory agencies around the world with regard to safety and efficacy and the drive by governments, insurers, and payers toward cost-disciplined medicinal therapy have caused the pharmaceutical industry as a whole to examine its processes, systems, and costs. Utilizing process improvement efforts such as Lean Sigma and technology advancements such as Process Analytical Technology (PAT), the pharmaceutical industry has risen to the challenge and is changing the development paradigm.

This issue of JPI contains articles and perspectives that are salient examples of both approaches. On the technology front, Tomoaki Sakamoto and co-workers report the utility of PAT to perform real-time analysis to effect quality control of a chemical reaction, while Claudia Corredor and researchers show that the combined use of computer simulation and automation can streamline analytical method development. Thomas Jennings describes a new and rapid alternative methodology for the determination of moisture in elastomer closures. Dan Dobry and colleagues provide a detailed overview of pharmaceutical spray drying, which shows promise as a means of developing bioavailable formulations for molecules with low solubility. Significant improvement in dissolution testing is reported by Scott Jennings and co-workers in their paper that describes a new design for the standard USP testing apparatus. Chedia Abdelkafi and colleagues provide a detailed perspective on the clinical trial supply chain process, which can be one of the costliest areas of pharmaceutical development. Finally, Donglei Mao and Qiang Zheng give a detailed overview of the sweeping regulatory changes taking place in China and offer perspective as to why many US and EU based pharmaceutical companies are investing heavily in this country.

As always, the Editorial Advisory Board seeks your thoughts, comments, and suggestions as to how to make JPI an even better journal and we look forward to hearing from you. In the meantime, enjoy the September issue.

S. Scypinski $(\bowtie)$

Bristol-Myers Squibb Company,

New Brunswick, NJ, USA

e-mail: Stephen.scypinski@bms.com 